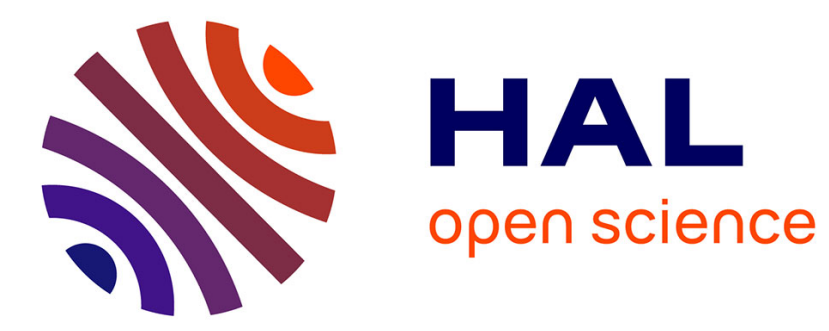

\title{
Transient pores in stretched vesicles: role of leak-out
} Françoise Brochard-Wyart, Pierre-Gilles de Gennes, Olivier Sandre

\section{To cite this version:}

Françoise Brochard-Wyart, Pierre-Gilles de Gennes, Olivier Sandre. Transient pores in stretched vesicles: role of leak-out. Physica A: Statistical Mechanics and its Applications, 2000, 278 (1-2), pp.32-51. 10.1016/S0378-4371(99)00559-2 . hal-02168814

\section{HAL Id: hal-02168814 https://hal.science/hal-02168814}

Submitted on 26 Aug 2019

HAL is a multi-disciplinary open access archive for the deposit and dissemination of scientific research documents, whether they are published or not. The documents may come from teaching and research institutions in France or abroad, or from public or private research centers.
L'archive ouverte pluridisciplinaire HAL, est destinée au dépôt et à la diffusion de documents scientifiques de niveau recherche, publiés ou non, émanant des établissements d'enseignement et de recherche français ou étrangers, des laboratoires publics ou privés. 


\title{
Transient pores in stretched vesicles : role of leak-out
}

\author{
F. Brochard-Wyart ${ }^{*}$, P.G. de Gennes ${ }^{\dagger}$ and O. Sandre ${ }^{*}$ \\ *Physico-Chimie Curie UMR 168 CNRS/Institut Curie \\ 11, rue Pierre et Marie Curie 75248 Paris Cedex 05 \\ ${ }^{\dagger}$ Physique de la Matière Condensée, Collège de France \\ 11, place Marcelin Berthelot 75231 Paris Cedex 05
}

PACS. : 87.22.Bt (Membrane and subcellular physics and structure), 87.45.-k (Biomechanics, biorheology, and biological fluid dynamics)

\begin{abstract}
We have visualized macroscopic transient pores in mechanically stretched giant vesicles. They can be observed only if the vesicles are prepared in a viscous solution to slow down the leak-out of the internal liquid. We study here theoretically the full dynamics of growth (driven by surface tension) and closure (driven by line tension) of these large pores. We write two coupled equations of the time evolution of the radii $r(t)$ of the hole and $R(t)$ of the vesicle, which both act on the release of the membrane tension. We find four periods in the life of a transient pore : (I) exponential growth of the young pore; (II) stop of the growth at a maximum radius $r_{m}$; (III) slow closure limited by the leak-out; (IV) fast closure below a critical radius, when leak-out becomes negligible. Ultimately the membrane is completely resealed.
\end{abstract}

Author manuscript of article published in Physica A, 2000. 278(1-2): 32-51

DOI: $\underline{10.1016 / S 0378-4371(99) 00559-2}$ 


\section{Introduction}

Forcing the passage of molecules, or genes, through a cellular membrane is a central problem of drug delivery. Model experiments based on vesicles represent a first step in this direction. A variety of physical techniques have been proposed to increase the permeability of lipid bilayers :

a) raising the osmotic pressure inside a vesicle induces a surface tension $\sigma$, and the permeability (of dyes, or of spin labels) is significantly increased [1]

b) if a bilayer becomes stuck on a porous surface (e.g. with a cationic bilayer and a negative surface) it tends to invade the pores; this again creates a tension $\sigma$ and an extra permeability [2]

c) instead of a porous medium it is possible to use a patched solid surface, some regions being attractive, and some being neutral or repulsive : a vesicle stuck on this surface becomes more permeable, or even breaks [3]

d) electroporation is a classical technique [4, 5] : with voltage drops of the order of 1 Volt across the bilayer, some large pores can be induced.

In a recent series of experiments [6] some of us have studied giant vesicles where the tension $\sigma$ could be established by two different ways :

(i) adhesion on an attractive surface, which generates flattened vesicles

(ii) irradiation of a vesicle (doped with suitable fluorescent dyes). For some (mysterious) reason, the equilibrium area per polar head under illumination is slightly reduced, and a tension $\sigma$ appears (within minutes). An effect of the same kind was reported for unlabeled vesicles submitted to laser tweezers [7]. This optically induced tension is very convenient, because it allows to work in a simple (spherical) geometry. 
Another important trick introduced in ref. [6] amounts to replace water by a more viscous mixture of water and glycerol (typical viscosity $\eta_{0}=30 \mathrm{cPoises}$ ). This allows to observe the formation, growth, and ultimate closure of large pores (radius $r \approx$ a few microns). The role of glycerol is explained in ref. [6] : a large pore allows for a certain leak-out of the inner compartment which has been known for some time [5, 8]. Leak-out reduces the tension $\sigma$, and leads to pore closure. But with glycerol this leak-out becomes very slow, and we can watch large pores persisting over long times (seconds). Some of the salient features of growth and closure in the slow leak-out limit have been modelized in ref. [6].

Our aim in the present paper is to make the theoretical discussion more general, in two directions :

a) we discuss fast leak-out as well as slow leak-out

b) it is assumed in ref. [6] that the growth and the late stages of closure were dominated by the internal viscosity $\eta_{2}$ of the lipid layer. This was correct for the experiments at hand, but in some other cases, all steps may be governed by the solvent viscosity $\eta_{0}$. This limit is discussed here in an appendix.

In section II we construct the dynamical equations describing the pore size $r(t)$ and the overall radius $R(t)$ of the vesicle - allowing for fast or slow leak-out. We display some numerical result on $r(t)$ for a few typical cases. In section III we concentrate on the slow leakout regime : here, it is possible to derive explicit formulas describing the four major time intervals involved : growth (I), bloom (II), leak-out (III) and ultimate closure (IV). Some of the physical questions which appear are discussed in section IV. 


\section{Transient pores}

\section{1) Counting the areas}

The geometry is shown on Fig. 1 . We constantly assume that the pore area $\left(\pi r^{2}\right)$ is much smaller than the overall area of the envelope sphere $\left(4 \pi R^{2}\right)$. We call $R_{i}$ the radius of the original vesicle under initial tension $\left(\sigma_{0}\right)$ and $R_{0}$ the radius in zero tension $(\sigma=0)$.

The lipid area $A_{L}$ differs from $4 \pi R_{0}^{2}$ because tension stretches our system :

$$
A_{L}=4 \pi R_{0}^{2}\left(1+\frac{\sigma}{E}\right)
$$

where $E$ is a two dimensional modulus. In the regime of interest here (weak tensions) $E$ is not related to the Van der Waals interactions in the lipid. It is in fact controlled by the unfolding of "wrinkles" (fluctuations) of the surface [9].

$$
E=\frac{48 \pi K_{b}}{R_{0}^{2}} \frac{K_{b}}{k T}
$$

where $K_{b}$ is the Helfrich bending constant [10] and $k T$ is the thermal energy. The overall distribution of areas is ruled by :

$$
4 \pi R^{2}=A_{L}+\pi r^{2}=4 \pi R_{0}^{2}\left(1+\frac{\sigma}{E}\right)+\pi r^{2}
$$

It is sometimes convenient to define a critical pore radius $r_{c}$ which corresponds to the complete relaxation of the tension $(\sigma=0)$ in situations of zero leakage $\left(R=R_{i}\right)$. Thus

$$
r_{c}^{2}=4 R_{0}^{2} \frac{\sigma_{0}}{E}
$$

\section{2) $\underline{\text { Rate equations }}$}

a) changes of the pore radius $r(t)$ are driven by two forces :

(i) the tension $\sigma$ (favoring expansion)

(ii) the line energy $\mathfrak{I}$ of the pore (favoring closure) 
When the internal viscosity of the bilayer is dominant [11], this leads to

$$
2 \eta_{2} d \frac{\dot{r}}{r}=\sigma-\frac{\mathfrak{J}}{r}
$$

where $d$ is the lipid thickness. In most macroscopic physical systems, $\mathfrak{I} / r$ is a negligible correction. But here it is important, because the tensions $\sigma$ are unusually small in vesicles.

b) Leak-out provides a way of changing the overall solvent volume, as explained in ref. $[5,6,8]$. The leak-out flux is

$$
Q=\text { const. } V_{L} r^{2}
$$

where $V_{L}$ is the leak-out velocity. The shear stresses involved in this outward flow are of order $\eta_{0} V_{L} / r$ where $\eta_{0}$ is the solvent viscosity. They are balanced by the Laplace pressure $p=2 \sigma / R$. The result is

$$
Q=\frac{2 \sigma}{3 \eta_{0} R} r^{3}=-4 \pi R^{2} \dot{R}
$$

On the whole, we now have three equations $(3,5,6)$ for three unknowns $(r, R, \sigma)$.

3) Non dimensional form

In the following, we consider small variations of the vesicle radius $R=R_{0}(1+\delta)$ and $R_{i}=R_{0}\left(1+\delta_{0}\right)$ at $t=0$, with $\delta, \delta_{0}<<1$. Then the critical pore radius introduced in Eq. (4) is given by $r_{c}^{2}=8 \delta_{0} R_{0}^{2}$. The two radii can be expressed in reduced units $: \tilde{r}=\frac{r}{r_{c}}$ and $\Delta=\frac{\delta_{0}-\delta}{\delta_{0}}$. This latter represents the drop of the vesicle radius due to leak-out : $\Delta$ varies from 0 to 1 as $R$ decreases from $R_{i}$ to $R_{0}$. The reduced time is $\tilde{t}=\frac{t}{\tau}$ with

$$
\frac{1}{\tau}=\frac{\sigma_{0}}{2 \eta_{2} d}
$$


Introducing the reduced surface tension $\tilde{\sigma}=\frac{\sigma}{\sigma_{0}}$, Eq. (3) rewrites

$$
\tilde{\sigma}=1-\tilde{r}^{2}-\Delta
$$

The differential equations $(5,6)$ are normalized as

$$
\left\{\begin{array}{l}
\frac{d \tilde{r}}{d \tilde{t}}=\tilde{r} \tilde{\sigma}-\tilde{\mathfrak{I}} \\
\frac{d \Delta}{d \tilde{t}}=\tilde{\sigma} \frac{\tilde{r}^{3}}{\tilde{r}_{L}}
\end{array}\right.
$$

The system (5', 6') contains only two adjustable parameters

$$
\tilde{\mathfrak{I}}=\frac{\mathfrak{I}}{\sigma_{0} r_{c}} \quad \text { (9) } \quad \text { and } \quad \tilde{r}_{L}=\frac{3 \pi \eta_{0}}{8 \eta_{2} d} \frac{R_{0}^{2}}{r_{c}}
$$

The boundary conditions are $\tilde{r}(\tilde{t}=0)=\tilde{r}_{i}$ and $\Delta(\tilde{t}=0)=0$.

The radius $r_{L}=\tilde{r}_{L} r_{c}$ is the upper pore size determined by leak-out first introduced in ref. [6]. The two extreme cases are (i) instantaneous release for $r_{L}=0$ : pore never opens (ii) gelified content for $\tilde{r}_{L} \rightarrow \infty$ : there is no leak-out at all, and $\Delta(\tilde{t})=0$ all the time. Then Eq. (5') reduces to $\frac{d \tilde{r}}{d \tilde{t}}=\tilde{r}\left(1-\tilde{r}^{2}\right)$ and can be solved analytically, as in [6] :

$$
\ln \frac{\tilde{r}}{\tilde{r}_{i}}-\frac{1}{2} \ln \frac{1-\tilde{r}^{2}}{1-\tilde{r}_{i}^{2}}=\tilde{t}
$$

\section{4) Numerical simulations}

In the following we take as a conjectural value $\tilde{r}_{i}=0.15$ for the initial radius, which comes from the fits of Eq. (11) with experimental data on vesicles that burst irreversibly [6]. We vary $\tilde{r}_{L}$ in a broad range $\left(10^{-2}\right.$ to $\left.10^{4}\right)$ as a model of the thickening of the ambient liquid. Simulations are performed using the simple Newton algorithm with a variable time step $\Delta \tilde{t}$ and an iteration number $N$ ranging from 4000 to 160000 for the largest $\tilde{r}_{L}$. We show here typical results : 
i) $\underline{\text { Fast leak-out }}\left(\tilde{r}_{L}<10\right)$

For the lowest values $\tilde{r}_{L}=10^{-2}-10^{-1}$, we see "aborted" pores with a dynamics in three stages only : growth (I), maximum (II) and fast closure (IV). The slow closure regime (III) appears clearly for $\tilde{r}_{L} \geq 1$, as seen on Fig. 2(a). It becomes broader when $\tilde{r}_{L}$ increases further. An intriguing result is that the final drop of the vesicle radius $\Delta_{\infty}$ (or equivalently the surface tension at end $\tilde{\sigma}_{\infty}$ ) is not a monotonous function of $\tilde{r}_{L}$. Instead, $\Delta_{\infty}$ has a maximum ( $\tilde{\sigma}_{\infty}$ has a minimum) near $\tilde{r}_{L}=10^{-1}$ : see Fig. 2(b), 2(c). A look to the curves of the outward flow on Fig. 2(d) helps to understand this. The peak value of $Q$ decreases monotonously with $\tilde{r}_{L}$, but the peak shape is not symmetrical : the left tail is lacking at $\tilde{r}_{L}=10^{-2}$, while there is a wide spread right tail for $\tilde{r}_{L} \geq 1$. The release of the vesicle content and the relaxation of surface tension being proportional to the integral of $Q$, they are both maximum at $\tilde{r}_{L}=10^{-1}$, which corresponds to a nearly symmetrical peak with maximum area. This means that there is a solvent viscosity that maximizes the delivery of the vesicle content : this effect could be useful for drug delivery by artificial vesicles and endocytosis processes involved in cells.

ii) $\underline{\text { Slow leak-out }}\left(\tilde{r}_{L}>>1\right)$

In that case, the slow closure (III) takes much more time than the three other stages of the long-lived pores. The closure velocity $V_{3}$ (defined as the slope of the curves on Fig. 3(a)) decreases when $\tilde{r}_{L}$ increases, for a given value of $\tilde{\mathfrak{I}}$. But the radius $\tilde{r}_{34}$ at the crossover between slow (III) and fast (IV) closures does not vary ( $\tilde{r}_{34} \approx 0.4$ on that example). The final value of $\Delta$ (and hence of the vesicle radius) is also constant, as seen on Fig. 3(b) : in the slow leak-out case, raising the solvent viscosity increases the pore lifetime, but it does not change the efficiency of the content release. However, one should keep in mind that Eq. (5) stands for 
a viscous dissipation dominated by the flows of lipid. For a very viscous content $\left(\tilde{r}_{L} \rightarrow \infty\right)$, a more appropriate model is developed in the Appendix.

iii) Influence of the line tension

The first role of the line energy $\widetilde{\mathfrak{I}}$ is to limit the pore nucleation. If $\tilde{\mathfrak{I}}>\tilde{r}_{i}$ (or $\left.\mathfrak{I}>\sigma_{0} r_{i}\right)$, the force acting on the pore edge is always negative, and it shrinks immediately. The other effects are studied by varying $\widetilde{\mathfrak{I}}$ among three values : $0.05,0.10$ and $0.14(<0.15)$.

We chose $\tilde{r}_{L}=5$, an intermediate value between fast and slow leak-out, which fits rather well our experimental results (that will be presented in a forthcoming paper). It is clear on the curves of Fig. 4 that, at higher line tension : (a) the pore reaches a smaller maximum radius $\tilde{r}_{m}$ and closes faster; (b) the decrease of the vesicle radius (and so the released volume) is smaller; (c) there is less relaxation of the surface tension at end; (d) the peak of the outward flow occurs at a later time. This last observation means that, for $\tilde{r}_{L}<10$, the line energy $\widetilde{J}$ plays a role even in the growth regime (I) : it slows down the pore opening which cannot be described simply by Eq. (11).

The two closure regimes (III) and (IV) depends also directly on $\tilde{\mathfrak{I}}$. The plot of the closure velocity (i.e. the negative values of $\dot{\tilde{r}}$ ) vs. pore radius (e) shows two parts : -a plateau between $\tilde{r}_{m}$ and $\tilde{r}_{34}$ corresponding to regime (III) at almost constant velocity denoted $V_{3}$-an acceleration of the closure, with a final velocity $\dot{\widetilde{r}}_{\infty}=\tilde{\mathfrak{I}}$. This fast regime (IV) will be discussed in the next section.

iv) Scaling laws (Fig. 5)

In order to compare the simulations to analytical formulas derived in section III, we analyze here the variation of some characteristic features of the curves with the two control parameters $\tilde{r}_{L}$ and $\tilde{\mathfrak{I}}$ : 
(a) the maximum pore radius $\tilde{r}_{m}$ vs. $\tilde{r}_{L}$

$\tilde{r}_{m}$ starts to increase with $\tilde{r}_{L}$, then it saturates at a level that depends on $\widetilde{\mathfrak{J}}$ only.

(b) the slow closure velocity vs. $\tilde{r}_{L}$

Although the pore radius $\tilde{r}(\tilde{t})$ appears linear in time during regime (III), a better linear regression is obtained when we plot $\ln \tilde{r}(\tilde{t})$ vs. $\tilde{t}$. The slope is then plotted as a function of $\tilde{r}_{L}$ for the three values of $\tilde{\mathfrak{J}}$. We find a scaling law that is valid from $\tilde{r}_{L}=10$ to $\tilde{r}_{L}=10^{4}$ (over three decades) :

$$
\left.\frac{d(\ln \tilde{r})}{d \tilde{t}}\right]_{(\mathrm{III})}=(0.53 \pm 0.3) \frac{\tilde{\mathfrak{I}}}{\widetilde{r}_{L}}
$$

For smaller $\tilde{r}_{L}$, this law overestimates the slope, which tends to a value that depends on $\tilde{\mathfrak{I}}$ only. Below $\tilde{r}_{L}=10^{-1}$, regime (III) is undistinguished from the final closure at velocity $\dot{\tilde{r}}_{\infty}=\widetilde{\mathfrak{I}}$ (the difference between $\widetilde{\mathfrak{I}}$ and the saturation levels on Fig. 5(b) comes from the semilogarithmic plot).

(c) the surface tension at the end $\tilde{\sigma}_{\infty}$ vs. $\tilde{\mathfrak{I}}$

For $\tilde{r}_{L}>10, \tilde{\sigma}_{\infty}$ is independent on $\tilde{r}_{L}$ and fits the scaling law $\tilde{\sigma}_{\infty}=1.89 \tilde{\Im}^{2 / 3}$.

In the following section, we derive analytical developments of the solutions $\tilde{r}(\tilde{t})$ for the "slow leak-out limit" $\tilde{r}_{L}>>1$. Interestingly, we have shown here numerically that, in practical, the condition $\tilde{r}_{L}>10$ is sufficient to ensure the validity of the scaling laws. 


\section{The slow leak-out limit}

We can solve the adimensional equations (5') and (6') in the limit of slow release $\tilde{r}_{L}>>1$. This condition simplifies the analytical resolution because the characteristic time of leak-out $\tau_{L} \cong \tilde{r}_{L} \tau$ is much larger than the rise time $\tau$. Therefore the initial growth of the pore is decoupled from the slow release of the encapsulated liquid. We analyze in this limit the four regimes of the life of a transient pore defined on the experimental curve (Fig. 1).

\section{$\underline{\text { Regime (I) : Growth }}$}

At time $t=0$, a pore nucleates at a radius $r_{i}$. This nucleation may be induced by a surface defect (dust particle, chemical heterogeneity, ...) or by thermal activation. The pore grows for $\tilde{r}_{i}>\widetilde{\mathfrak{J}}$. At short times, we have the following approximations :

$$
\left\{\begin{array} { l } 
{ \Delta = 0 } \\
{ \tilde { \sigma } = 1 }
\end{array} \quad \text { or with dimensional units } \quad \left\{\begin{array}{l}
R=R_{i} \\
\sigma=\sigma_{0}
\end{array}\right.\right.
$$

Eq. (5') then reduces to $\frac{d \tilde{r}}{d \tilde{t}}=\tilde{r}$, i.e. :

$$
\tilde{r} \cong \tilde{r}_{i} e^{\tilde{t}} \quad r \cong r_{i} e^{t / \tau}
$$

In the limit $\tilde{r}_{L} \gg 1$, the solution over the whole regime I verifies $\Delta=0$, and the pore radius is given by Eq. (11) as in the case $\tilde{r}_{L} \rightarrow \infty$.

\section{$\underline{\text { Regime (II) : Maximum radius }}$}

The hole grows up to $\tilde{r}_{m}$, and starts to decrease. At its maximum size, $\dot{\widetilde{r}}_{m}=0$ leads to a first relation :

$$
\tilde{r}_{m} \tilde{\sigma}_{m}=\tilde{\mathfrak{I}} \quad r_{m} \sigma_{m}=\mathfrak{I}
$$

where $\tilde{\sigma}_{m}=1-\tilde{r}_{m}^{2}-\Delta_{m}$. We can estimate $\Delta_{m}$ by using a scaling solution of Eq. (6’) :

$$
\Delta_{m} \cong \tilde{r}_{m}^{3} \frac{\tilde{\sigma}_{m}}{\tilde{r}_{L}}=\tilde{r}_{m}^{2} \frac{\tilde{\mathfrak{I}}}{\tilde{r}_{L}} \quad R_{i}^{2}-R_{m}^{2} \cong 4 r_{m}^{2} \frac{\mathfrak{I}}{\sigma_{0} r_{L}}
$$


In the limit $\tilde{r}_{L}>>1, \tilde{r}_{m}$ becomes close to one, and $\Delta_{m}<<1$. Eq. (15) leads to :

$$
\begin{aligned}
& \tilde{\Im} \cong \tilde{r}_{m}\left(1-\tilde{r}_{m}^{2}\right) \\
& \text { i.e. } \quad \tilde{r}_{m} \cong 1-\frac{\tilde{J}}{2} \quad r_{m} \cong r_{c}-\frac{\mathfrak{I}}{2 \sigma_{0}}
\end{aligned}
$$

The parameter $\mathfrak{I} / \sigma_{0}$ has a simple meaning : it is the nucleation radius of a pore (in conditions of ideal nucleation) as derived from a pore energy $\pi r^{2} \sigma_{0}-2 \pi r \mathfrak{I}$. Indeed, this radius is small $(<1 \mu \mathrm{m})$.

The numerical simulations show that the maximum pore radius fits well Eq. (17) as soon as $\tilde{r}_{L}>20$ with less than $1 \%$ uncertainty : Fig. 5(a). The linearized form (18) becomes a good approximation for small values of $\tilde{\mathfrak{J}}$.

The curvature $\ddot{\widetilde{r}}_{m}=-\tilde{r}_{m} \dot{\Delta}_{m}=-\tilde{r}_{m}^{4} \frac{\tilde{\sigma}_{m}}{\tilde{r}_{L}}=-\frac{\tilde{r}_{m}^{3}}{\widetilde{r}_{L}} \tilde{\mathfrak{I}}$ tends to zero like $\tilde{r}_{L}^{-1}$ when $\tilde{r}_{L} \rightarrow \infty$.

\section{Regime (III) : Quasistatic leak-out $\left(r_{23}>r>r_{34}\right)$}

As $r(t)$ starts to decrease, $\sigma(r, R)$ grows : a release of the inner liquid is then necessary in order to maintain an almost zero surface tension. More precisely, the pore closes if the driving force is negative, i.e. :

$$
\tilde{r} \tilde{\sigma}<\tilde{\mathfrak{I}} \quad r \sigma<\mathfrak{I}
$$

We shall see that the inequality (19) is very nearly an equality : in regime (III) the driving force of Eq. (5) is nearly zero $\sigma \cong \mathfrak{J} / r$ or

$$
\frac{\sigma-\mathfrak{I} / r}{\sigma}<<1
$$

We may say the regime (III) is quasistatic. A detailed argument for this follows :

We calculate the derivative of Eq. (5') :

$$
\ddot{\widetilde{r}}=\dot{\widetilde{r}}\left(1-\tilde{r}^{2}-\Delta\right)-\tilde{r}(2 \tilde{r} \dot{\widetilde{r}}+\dot{\Delta})
$$


In this regime, the closure velocity is very slow, and we can assume $\tilde{r} \tilde{\sigma} \cong \widetilde{\mathfrak{J}}$ to evaluate $\dot{\Delta} \cong \frac{\tilde{r}^{2}}{\widetilde{r}_{L}} \widetilde{\Im}$. Eq. (21) becomes :

$$
\ddot{\widetilde{r}} \cong \dot{\widetilde{r}}\left(\frac{\tilde{\mathfrak{J}}}{\widetilde{r}}-2 \tilde{r}^{2}\right)-\frac{\tilde{r}^{3}}{\widetilde{r}_{L}} \tilde{\mathfrak{I}} \cong 0
$$

We set $\ddot{\tilde{r}}=0$ because the scale time variation for leak-out is $\tilde{r}_{L}$ times larger $\left(\frac{\ddot{\tilde{r}}}{\dot{\tilde{r}}} \cong \frac{1}{\widetilde{r}_{L}}<<1\right)$ : the closure is at almost constant velocity. If $\widetilde{\mathfrak{J}}<<2 \tilde{\mathrm{r}}^{3}$ (or $r>r_{34}$, which defines the limit of regime (III), where the pore is not too small so that leak-out has a significant effect), the first term between parentheses in Eq. (22) is negligible. This leads to :

$$
2 \dot{\widetilde{r}}+\frac{\tilde{r}}{\widetilde{r}_{L}} \tilde{\Im} \cong 0
$$

Introducing $r_{23}$ and $t_{23}$ as the cross-over values between regimes (II) and (III), the solution is :

$$
\ln \frac{\tilde{r}}{\widetilde{r}_{23}}=-\frac{\tilde{\mathfrak{I}}}{2 \tilde{r}_{L}}\left(\tilde{t}-\tilde{t}_{23}\right) \quad \ln \frac{r}{r_{23}}=-\frac{2 \mathfrak{I}}{3 \pi \eta_{0} R_{0}^{2}}\left(t-t_{23}\right)
$$

This formula is very closed to the scaling law deduced from Fig. 5(b). A linearized form of Eq. (24) is $\tilde{r} \cong \widetilde{r}_{23}\left[1-\frac{\tilde{\mathfrak{J}}}{2 \widetilde{r}_{L}}\left(\tilde{t}-\tilde{t}_{23}\right)\right]$. Taking into account that $r_{23} \cong r_{m}$, we find an approximate expression for the closure velocity during regime (III) :

$$
V_{3} \cong \frac{2 \Im r_{m}}{3 \pi \eta_{0} R_{0}^{2}}
$$

Regime (IV) : Fast closure $\left(r<r_{34}\right)$

When the size of the hole becomes too small, leak-out is nearly suppressed. The radius $r_{34}$ at cross-over between regimes (III) and (IV) has already been introduced, and verifies : 


$$
2 \tilde{r}_{34}^{3} \cong \widetilde{\mathfrak{I}} \quad 2 r_{34}^{3} \cong \frac{\Im r_{c}^{2}}{\sigma_{0}}
$$

The vesicle has almost reached a constant radius $R_{\infty}$ and Eqs. $\left(5^{\prime}, 6^{\prime}\right)$ becomes :

$$
\left\{\begin{array}{l}
\dot{\tilde{r}}=\tilde{r}\left(1-\tilde{r}^{2}-\Delta_{\infty}\right)-\tilde{\Im} \\
\dot{\Delta} \cong 0
\end{array}\right.
$$

Assuming that $\tilde{r} \tilde{\sigma} \cong \widetilde{\mathfrak{J}}$ is still valid at the cross-over enables to estimate the surface tension at the end :

$$
\tilde{\sigma}_{\infty}=1-\Delta_{\infty} \cong \frac{\widetilde{\mathfrak{I}}}{\widetilde{r}_{34}}+\widetilde{r}_{34}^{2} \cong 3 \tilde{r}_{34}^{2} \cong 1.89 \widetilde{\mathfrak{I}}^{2 / 3}
$$

This relation (also verified on the numerical simulations) shows that the vesicle recovers a non-negligible surface tension at the end of the pore closure. For example above $40 \%$ of the initial tension is maintained for $\widetilde{\mathfrak{I}}=0.10$. This could explain why series of successive pores are observed in the experiments.

The fast closure of the pore is governed by Eq. (27), which rewrites :

$$
\dot{\tilde{r}}=\tilde{r}\left(3 \tilde{r}_{34}^{2}-\tilde{r}^{2}\right)-2 \tilde{r}_{34}^{3}
$$

We do not propose an analytical solution that would be valid for the whole duration of regime (IV). Instead, we just look at the final stage, when the equation is linear :

$$
\dot{\tilde{r}} \cong 3 \widetilde{r}_{34}^{2}\left(\tilde{r}-\frac{2}{3} \widetilde{r}_{34}\right)
$$

This is a good approximation after a time $t_{4}$ that is defined arbitrarily such as

$$
\tilde{r}\left(\tilde{t}_{4}\right)=\frac{\tilde{r}_{34}}{3}
$$

Then the integration leads to :

$$
\ln \left(1-\frac{3 \tilde{r}}{2 \tilde{r}_{34}}\right)=3 \tilde{r}_{34}^{2}\left(\tilde{t}-\tilde{t}_{4}\right)-\ln 2
$$

which can be linearized as 


$$
\tilde{r} \cong \frac{2 \ln 2}{3} \tilde{r}_{34}-\tilde{\Im}\left(\tilde{t}-\tilde{t}_{4}\right)
$$

Therefore it appears that the final closure of the pore is at constant velocity :

$$
V_{4} \cong \frac{\mathfrak{I}}{2 \eta_{2} d}
$$

This linear regime is valid over a range that is not negligible, since $\tilde{r}\left(\tilde{t}_{4}\right) \cong 0.26 \widetilde{\mathfrak{I}}^{1 / 3}$ (for example $r\left(t_{4}\right) \cong 0.12 r_{c}$ for $\tilde{\mathfrak{I}}=0.10$ ).

\section{Conclusions}

1) A thickening of the solution increases the size of transient pores. The cross-over between "fast" and "slow" leak-out is ruled by $r_{L} \cong \frac{\eta_{0} R_{0}^{2}}{\eta_{2} d}$. If $\tilde{r}_{L}=r_{L} / r_{c}<1$, the blooming of pores (regime II) occurs for a radius $r_{m} \cong r_{L}$ : pores abort before reaching the equilibrium size $r_{c}$, because enough surface tension is relaxed by the fast release of the vesicle content. If $\tilde{r}_{L}>1$, a full blooming of pores is allowed : slowing down leak-out leads to mature pores, now at almost their equilibrium radius. After blooming, pores close slowly (regime III), before dying abruptly (regime IV). In the limit of slow leak-out $\left(\tilde{r}_{L}>>1\right)$, the dynamical equation for $r(t)$ can be derived by a simple scaling argument. Line tension closes the pore with a net power that equilibrates the entropy production per unit time :

$$
-\mathfrak{I} \dot{r}=T \dot{S}=\eta_{0}\left(\frac{V_{L}}{r}\right)^{2} r^{3}
$$

The viscous losses are due to flows of solution through the pore. The leak-out velocity has already been estimated as $V_{L}=\frac{r \sigma}{\eta_{0} R}$. The closure is quasi-stationary and we can use approximation (20) to get $V_{L} \cong \frac{\mathfrak{I}}{\eta_{0} R}$. Thus Eq. (36) rewrites 


$$
-\mathfrak{\Im} \dot{r}=\frac{\mathfrak{J}^{2}}{\eta_{0} R^{2}} r
$$

This simple expression contains the salient features of regime (III) : the logarithm of the pore radius decreases linearly in time, with a slope in agreement with Eq. (24). Of course the exact prefactor cannot be obtained by this scaling argument.

2) Looking back at Fig. 1, our theoretical picture allows us to derive with a good accuracy two important parameters of the membrane: the surface viscosity $\eta_{s}=\eta_{2} d$ and the line energy of a pore. Indeed the ratio between the velocities in the two linear regimes (III) and (IV) contains $\eta_{s}$ as the only unknown :

$$
\frac{V_{4}}{V_{3}}=\frac{3 \pi R_{0}^{2}}{4 r_{m}} \frac{\eta_{0}}{\eta_{s}}
$$

The values $R_{0}=14.5 \mu \mathrm{m}, r_{m}=4.5 \mu \mathrm{m}, \eta_{0}=3.2 \times 10^{-2} \mathrm{Nsm}^{-2}$ (=32cPoise), $V_{3}=0.9 \mu \mathrm{m} / \mathrm{s}$ and $V_{4} \approx 9 \mu \mathrm{m} / \mathrm{s}$ are measured. Thus we calculate $\eta_{s} \approx 3.5 \times 10^{-7} \mathrm{Nsm}^{-1}\left(=3.5 \times 10^{-4}\right.$ surfacePoise $)$. The thickness of a phospholipid bilayer being $d \approx 3.5 \mathrm{~nm}$, we predict the bulk viscosity of 1,2 dioleoyl-sn-glycero-3-phosphocholine (DOPC) : $\eta_{2} \approx 100 \mathrm{Nsm}^{-2}\left(=10^{5} \mathrm{cPoise}\right)$. We calculate also (from $V_{3}$ only) $\mathfrak{I} \approx 0.6 \times 10^{-11} \mathrm{~N}$. The usual measurements of line energies $\mathfrak{I}$ are extremely delicate $[4,5,8,12]$, and $\mathfrak{I}$ is expected to be sensitive to certain lipid impurities. We have studied one extreme case, based on the well-known effect of cholesterol to stiffen the bilayers. Our preliminary results show that the line energy is three times larger for a membrane containing DOPC and 30mol\% cholesterol.

3) The presence of glycerol may modify the membrane characteristics and the twodimensional mobility of lipids. Vesicles of DOPC containing a hydrophobic fluorophore were studied by time-resolved fluorescence spectroscopy [13]. The rotational motion of the molecular probe was slowed down at high glycerol content (in a 91:9 w/w mixture of glycerol and water, the rotation time becomes three times larger than in water). This result tells us that 
a slight viscous thickening of the lipid is observed, but no dramatic effects are reported. This is crucial because the maximum size of transient pores in the fast leak-out limit is $r_{L} \cong \frac{\eta_{0}}{\eta_{s}} R_{0}^{2}$. If we assume no dependence of $\eta_{s}$ on the glycerol content, the size of transient pores in pure water are expected to be thirty times smaller : 100-200nm. These pores open and close before reaching a visible size in optical microscopy. But their presence explains certain anomalous permeabilities observed for stretched adhesive vesicles [14]. They have also been observed in the membrane of human erythrocytes [15]. The authors used a very clever setup to freeze the cells with a controlled time delay after electroporation, and observe them later by electron microscopy. Despite statistical scatter among the cells, they reached a good insight of the pore dynamics. They divided it into three stages : (i) within 3ms, pores expand rapidly up to 20$40 \mathrm{~nm}$ in diameter (ii) between $20 \mathrm{~ms}$ and $220 \mathrm{~ms}$ they saturate at a maximum diameter of 100 160nm (iii) after 1s to $5 \mathrm{~s}$ almost all the pores have shrank. This scenario looks similar to ours except that the scales of times and pore sizes are about thirty times smaller.

4) We assumed that, at all times, $\sigma$ is uniform all over the vesicle. This means that no rim surrounds the hole. This is the signature of plug flows extending over large distances, which have been observed in the bursting of polymer "bare" films (i.e. with no surfactants, [11]). An experimental evidence of this assumption is that no bright circle around the pore is visible in fluorescence microscopy. This simplification may be lost when the membrane viscosity is no more dominant. Our experiments on liquid/liquid dewetting have shown that indeed a rim appears when dissipation in the bulk liquid overcomes dissipation in the film [16].

Note added in proof: While this paper was in review, we became aware that Eq. (15) in H. Isambert, Phys. Rev. Lett. 80 (1998) 3404-3407 is an equivalent form of our Eq. (11) for the pore radius vs. time in the case of negligible leak-out. 


\section{Appendix : transient pores in low viscosity membranes}

We now study the opposite limit, when the viscous dissipation in the membrane is small compared to the dissipation of the backflows induced by the pore opening in the surrounding liquid. The extension of Eq. (5), incorporating a mixed dissipation both in the membrane and in the solvent may be written as

$$
\eta_{0} r \dot{r}+2 \eta_{2} d \dot{r}=r \sigma-\mathfrak{J}
$$

The first term is the contribution of the viscous dissipation in the solvent. It can be justified by the following scaling argument. The entropy production per unit time is $T \dot{S} \cong \eta_{0}\left(\frac{\dot{r}}{r}\right)^{2} r^{3}$, where $r^{3}$ is the volume of the flows occurring through the pore. The transfer of surface and line energies into viscous losses leads to Eq. (A1). Notice that for the backflows in the solvent, we have only a scaling form and we do not know the exact numerical coefficient. This is not true for the second term, because dissipation due to radial plug flows in the membrane was calculated exactly.

We study here the case where solvent dissipation is dominant. This implies $\eta_{0} r>>\eta_{2} d$. Eq. (A1) then becomes

$$
\begin{gathered}
\eta_{0} r \dot{r}=r \sigma-\mathfrak{I} \\
\text { where } \sigma=\sigma_{0}\left(1-\frac{r^{2}}{r_{c}^{2}}-\frac{R_{i}^{2}-R^{2}}{R_{i}^{2}-R_{0}^{2}}\right)
\end{gathered}
$$

The other rate equation (describing leak-out) is not modified

$$
-4 \pi R^{2} \dot{R}=\frac{2 \sigma}{3 \eta_{0} R} r^{3}
$$

Eqs. (A2, A3) with initial conditions $r(t=0)=r_{i}>\frac{\mathfrak{I}}{\sigma_{0}}$ and $R(t=0)=R_{i}$ describes the full life of transient pores in that limit. At short times the pores grow at constant velocity 
$\dot{r}_{g}=\frac{\sigma_{0}}{\eta_{0}}$, where $\sigma_{0}$ is the stress before hole opening. We can define a characteristic time of growth

$$
\tau^{\prime}=\frac{r_{c}}{\dot{r}_{g}}=\frac{r_{c} \eta_{0}}{\sigma_{0}}
$$

Eqs. (A2, A3) can be written in adimensional units $\tilde{r}=\frac{r}{r_{c}}, \tilde{t}^{\prime}=\frac{t}{\tau^{\prime}}, \Delta=\frac{\delta_{0}-\delta}{\delta_{0}}$ and $\tilde{\sigma}=\frac{\sigma}{\sigma_{0}}$ as $\quad\left\{\begin{array}{l}\tilde{r} \frac{d \tilde{r}}{d \tilde{t}^{\prime}}=\tilde{r} \tilde{\sigma}-\tilde{J} \\ \tilde{\sigma}=1-\tilde{r}^{2}-\Delta \\ \frac{d \Delta}{d \tilde{t}^{\prime}}=\tilde{\sigma} \frac{\tilde{r}^{3}}{r_{L}^{\prime}}\end{array}\right.$

where $\quad \tilde{\mathfrak{I}}=\frac{\mathfrak{I}}{\sigma_{0} r_{c}} \quad$ and $\quad r_{L}{ }^{\prime}=\frac{R_{0}^{2}}{r_{c}^{2}}$

Because the same viscosity is involved in the pore expansion and in solvent release, $\eta_{0}$ does not show up in the controlled parameter. In addition the pore can reseal only if $r_{c}<R_{0}$ (otherwise the vesicle bursts irreversibly). Therefore in practice $r_{L}{ }^{\prime}>1$, which means that when dissipation in backflows dominates, the pores are always in the slow leak-out regime. Pores will open at constant velocity $\dot{r}_{g}$ (I) up to a large size $r_{m}$ (II). Then they close slowly during step (III) which is identical to the previous case, with a closure velocity $V_{3} \cong \frac{2 \mathfrak{I} r_{m}}{3 \pi \eta_{0} R_{0}^{2}}$. The cross-over radius $r_{34}$ between slow and fast closure is unchanged. But the fast regime (IV) is entirely different. Eq. (A1) shows that $r^{2} \cong \frac{\mathfrak{I}}{\eta_{0}}\left(t_{\infty}-t\right)$, i.e. the slope as $r \rightarrow 0$ becomes infinite. The line tension can still be deduced, from the curvature of $r(t)$ near $r=0$. Numerical simulations of Eq. (A5) are shown in Fig. 6 for several values of $r_{L}{ }^{\prime}(=5,10,50)$. 


\section{Glossary}

\begin{tabular}{|c|c|c|c|}
\hline$r$ & pore radius & $\eta_{2}$ & lipid viscosity \\
\hline$r_{i}$ & pore radius at nucleation & $\eta_{s}$ & surface viscosity \\
\hline$r_{c}$ & pore radius at zero tension & $\eta_{0}$ & viscosity of solution \\
\hline$R$ & vesicle radius & $V_{L}$ & leak-out velocity \\
\hline$R_{i}$ & initial vesicle radius & $Q$ & leak-out flux \\
\hline$R_{0}$ & vesicle radius at zero tension & $r_{L}$ & $\begin{array}{l}\text { characteristic radius of leak- } \\
\text { out }\end{array}$ \\
\hline$K_{b}$ & Helfrich bending constant & $\tau$ & rise time of pore growth (I) \\
\hline$E$ & surface stretching modulus & $r_{m}$ & radius at maxium (II) \\
\hline$d$ & membrane thickness & $r_{23}$ & $\begin{array}{l}\text { pore radius at cross-over } \\
\text { between (II) and (III) }\end{array}$ \\
\hline $\mathfrak{I}$ & line tension & $V_{3}$ & $\begin{array}{l}\text { slow closure velocity limited } \\
\text { by leak-out (III) }\end{array}$ \\
\hline$\sigma$ & surface tension & $r_{34}$ & $\begin{array}{l}\text { pore radius at cross-over } \\
\text { between (III) and (IV) }\end{array}$ \\
\hline$\sigma_{0}$ & $\begin{array}{l}\text { surface tension before pore } \\
\text { opening }\end{array}$ & $V_{4}$ & $\begin{array}{l}\text { fast closure velocity at end } \\
\text { (IV) }\end{array}$ \\
\hline
\end{tabular}




\section{References}

[1] M. Dvolaïtsky, P.G. de Gennes, M-A. Guedeau, L. Jullien (1993) C. R. Acad. Sci. Paris 316 II 1687-1690.

[2] M-A. Guedeau-Boudeville, L. Jullien, J-M. di Miglio (1995), communicated by P.G. de Gennes, Proc. Natl. Ac. Sc. USA 92 1-3.

[3]. A-L. Bernard, M-A. Guedeau-Boudeville, O. Sandre, S. Palacin, J-M. di Miglio, L. Jullien (2000), Langmuir 16 6801-6808

[4] W. Harbich, W. Helfrich (1979) Z. Naturforsch. 34a 1063-1065.

[5] D.V. Zhelev, D. Needham (1993) Biochim. Biophys. Acta 1147 89-104.

[6] O. Sandre, L. Moreaux, F. Brochard-Wyart (1999), communicated by P.G. de Gennes, Proc. Natl. Ac. Sc. USA 96 10591-10596. Commentary by P. Pincus p10550.

[7] R. Bar-Ziv, T. Frisch, E. Moses (1995) Phys. Rev. Lett. 75, 3481-3484.

[8] J. Moroz, P. Nelson (1997) Biophys. J. 72 2211-2216.

[9] F. Brochard, P.G. de Gennes, P.Pfeuty (1976) J. Phys. (France) 37 1099-1103.

[10] W. Helfrich (1973) Z. Naturforsch. 28c 693-703.

[11] G. Debrégeas, P. Martin, F. Brochard-Wyart (1995) Phys. Rev. Lett. 75, 3886-3889

[12] L.V. Chernomordik, M.M. Kozlov, G.B. Melikyan, I.G. Abidor, V.S. Markin, Y.A. Chizmadzhev (1985) Biochim. Biophys. Acta 812 643-655.

[13] L. B-Å Johansson, B. Kalman, G. Wikander, A. Fransson, K. Fontell, B. Bergenstål, G. Lindblom (1993) Biochim. Biophys. Acta 1149 285-291.

[14] A-L. Bernard, M-A. Guedeau-Boudeville, J-M. di Miglio, L. Jullien (2000) Langmuir 16 6809-6820.

[15] D.C. Chang (1992) in Guide to Electroporation and Electrofusion, Chang, Chassy, Saunders, Sowers Eds. (Acad. Press. San Diego) p9-27; D.C. Chang (1990) Biophys. J. 58 112.

[16] F. Brochard-Wyart, G. Debregeas, R. Fondecave, P. Martin (1997) Macromolecules 30 1211-1213. 


\section{Figures and captions}
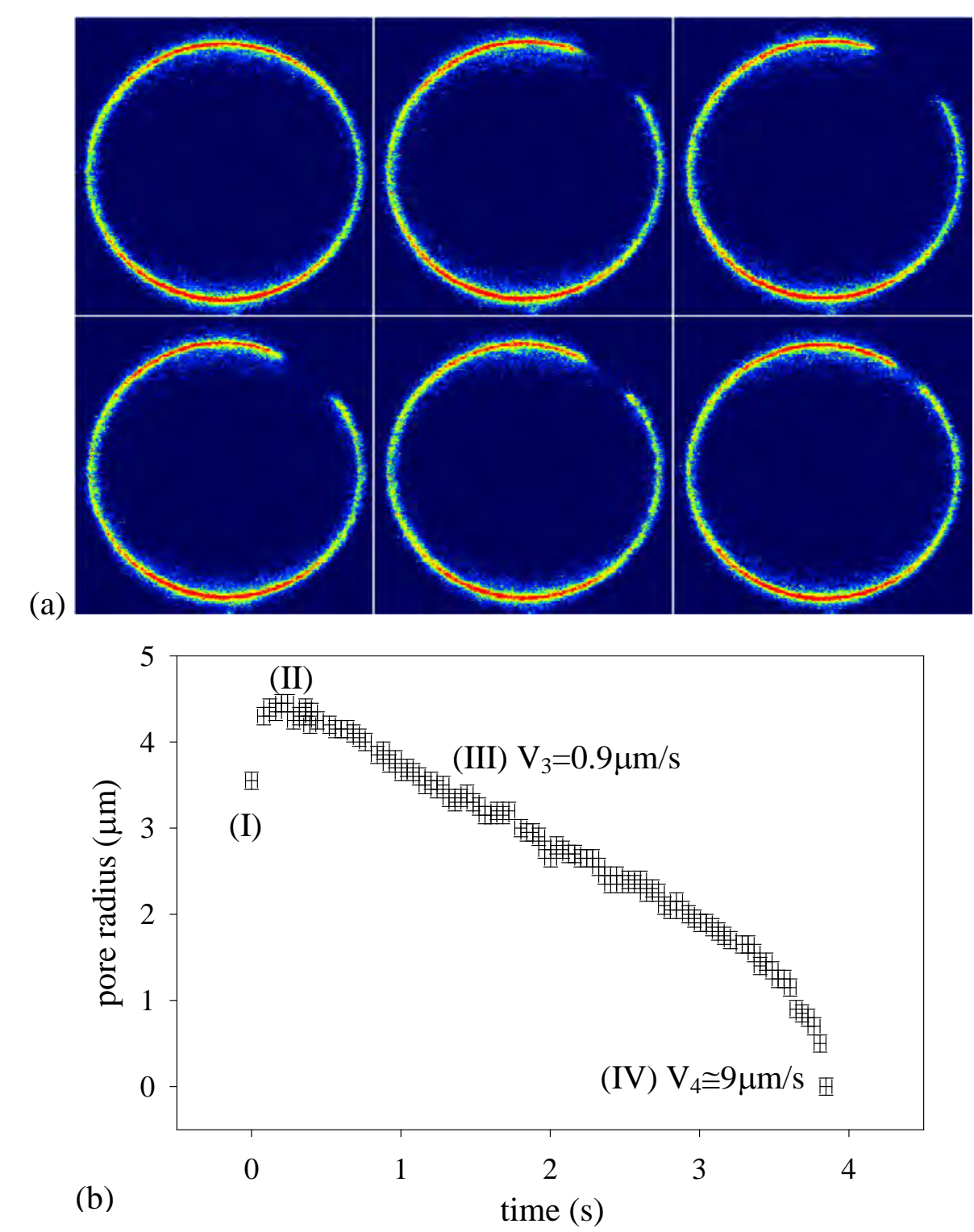

Fig. 1 : Typical experiment (a) time sequences of a transient pore in a giant vesicle stretched by intense illumination; the membrane lipid is DOPC labeled as described in ref. [6]; the solvent is a 71:29 w/w mixture of glycerol and water, with a measured viscosity $\eta_{0}=32 \pm 0.4 \mathrm{cPoise}$; the solutes are sucrose $0.1 \mathrm{M}$ inside, glucose $0.1 \mathrm{M}$ outside; (b) corresponding measurement of pore radius vs. time, showing four stages in the pore dynamics. 
(a)

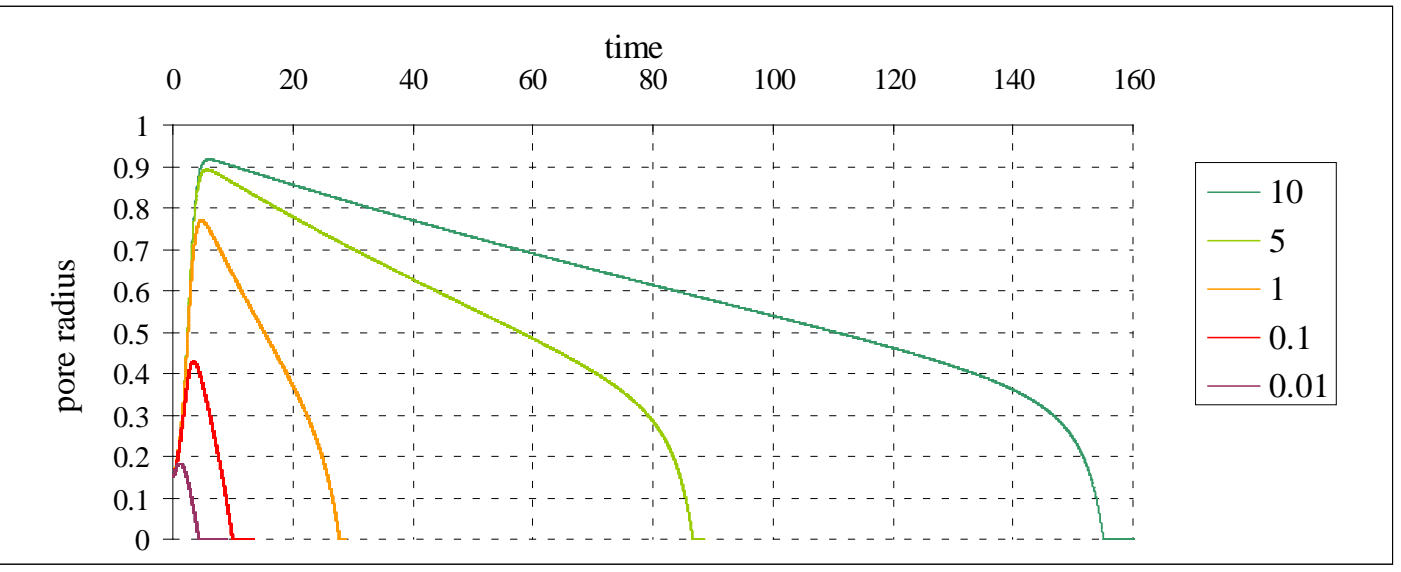

(b)

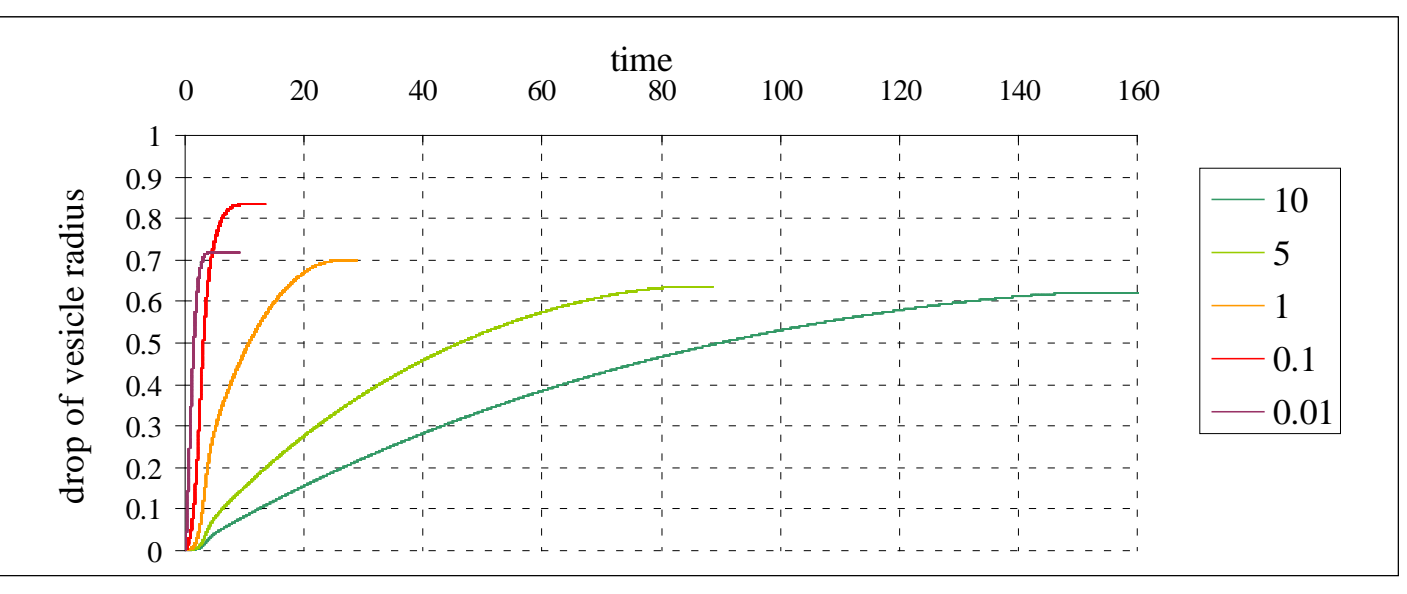

(c)

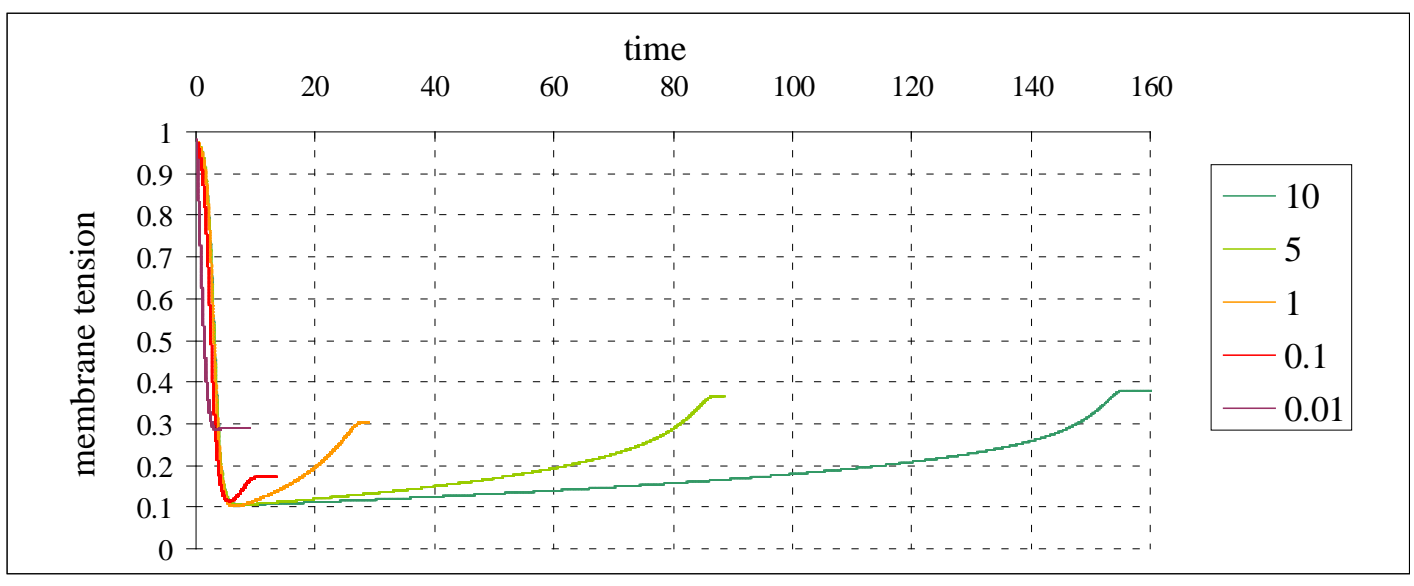

(d)

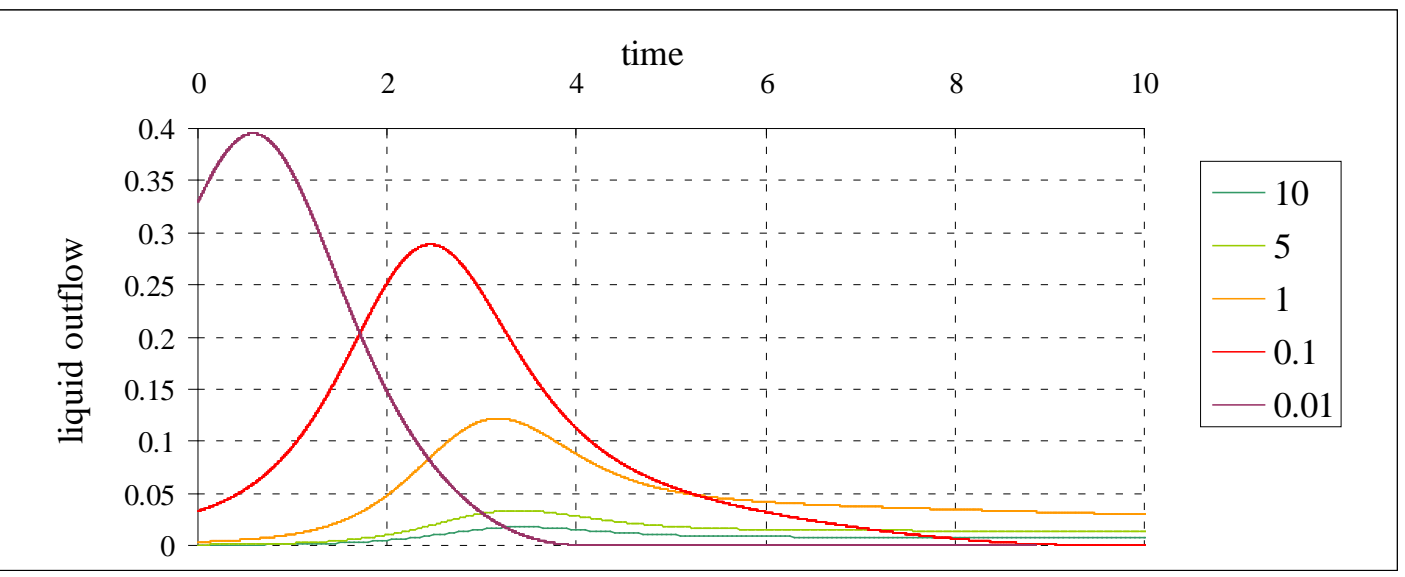


Fig. 2 : Fast leak-out regime - numerical simulations of Eqs. (5', 6') for constant line tension $\widetilde{\mathfrak{I}}=0.1$ and different low values of the leak-out parameter $\tilde{r}_{L}=0.01-0.1-1-5-10$; (a) pore radius $\tilde{r}$ vs. time $\tilde{t}$; (b) drop of vesicle radius $\Delta$ vs. time $\tilde{t}$; (c) surface tension $\tilde{\sigma}$ vs. time $\tilde{t}$; (d) liquid outflow $Q$ in the early times.

(a)
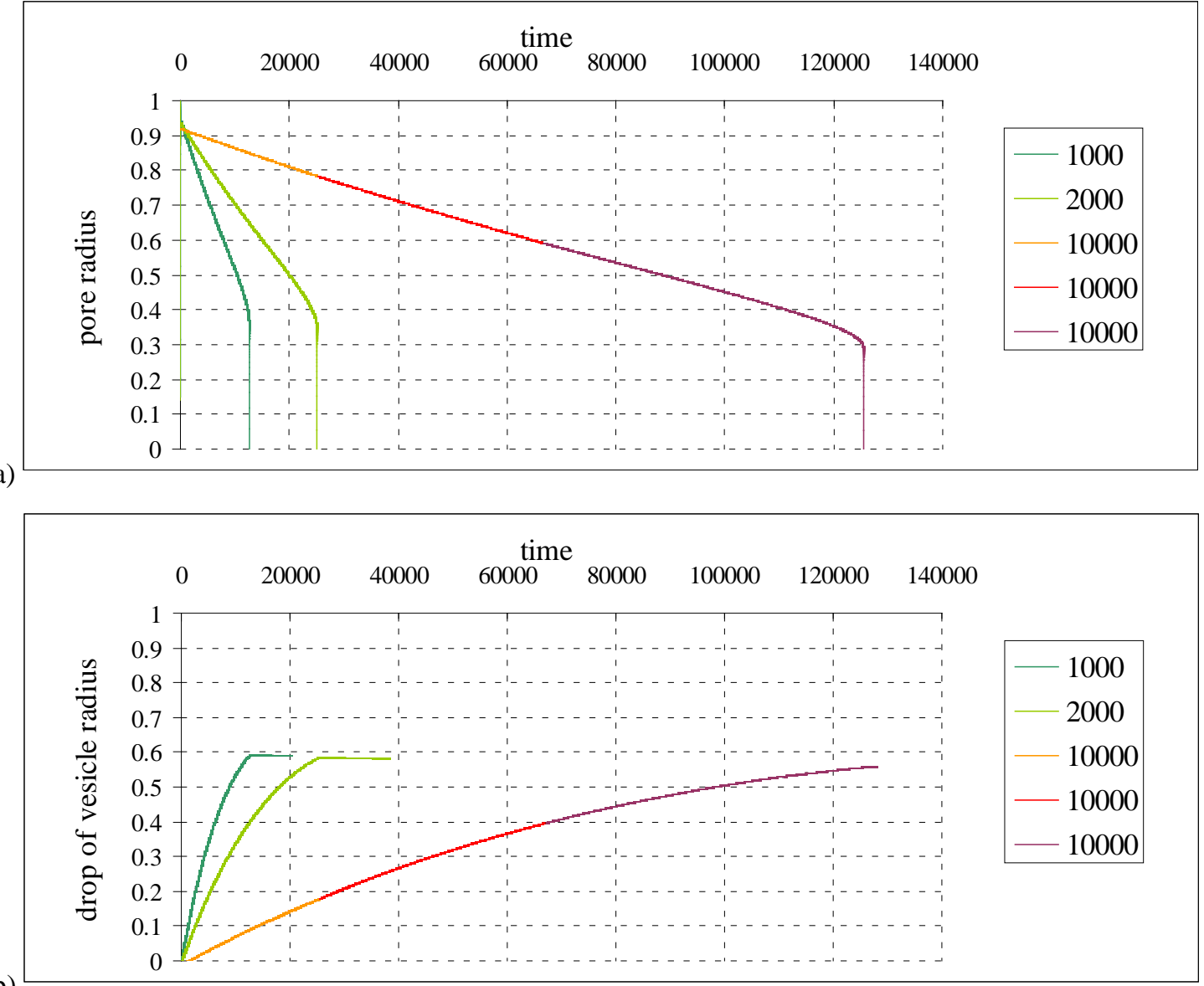

Fig. 3 : Slow leak-out regime - numerical simulations of Eqs. (5', 6’) for constant line tension $\widetilde{\mathfrak{I}}=0.1$ and different high values of the leak-out parameter $\tilde{r}_{L}=10^{3}-2 \times 10^{3}-10^{4}$; (a) pore radius $\tilde{r}$ vs. time $\tilde{t}$; (b) drop of vesicle radius $\Delta$ vs. time $\tilde{t}$. 
(a)

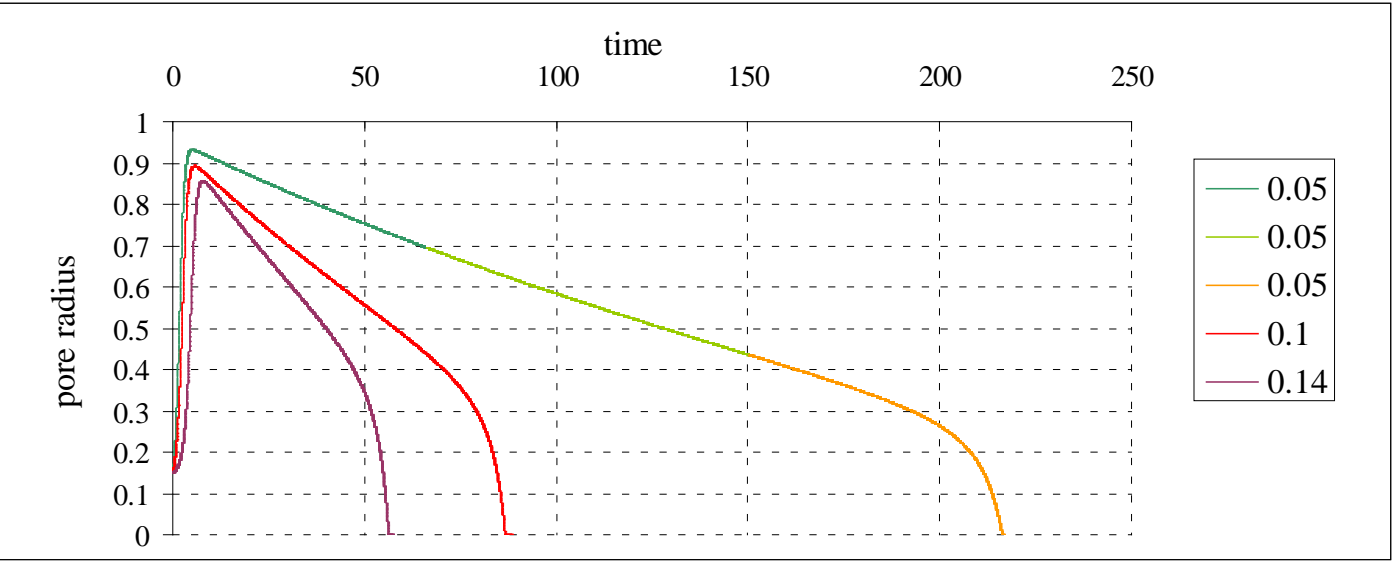

(b)

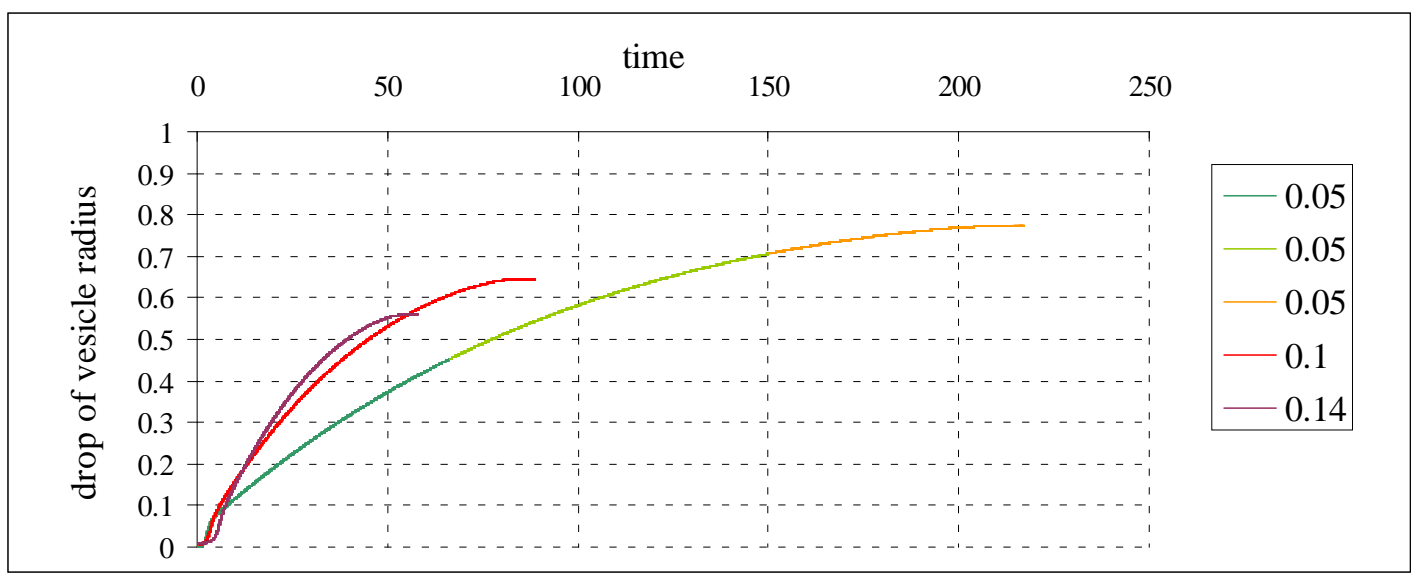

(c)

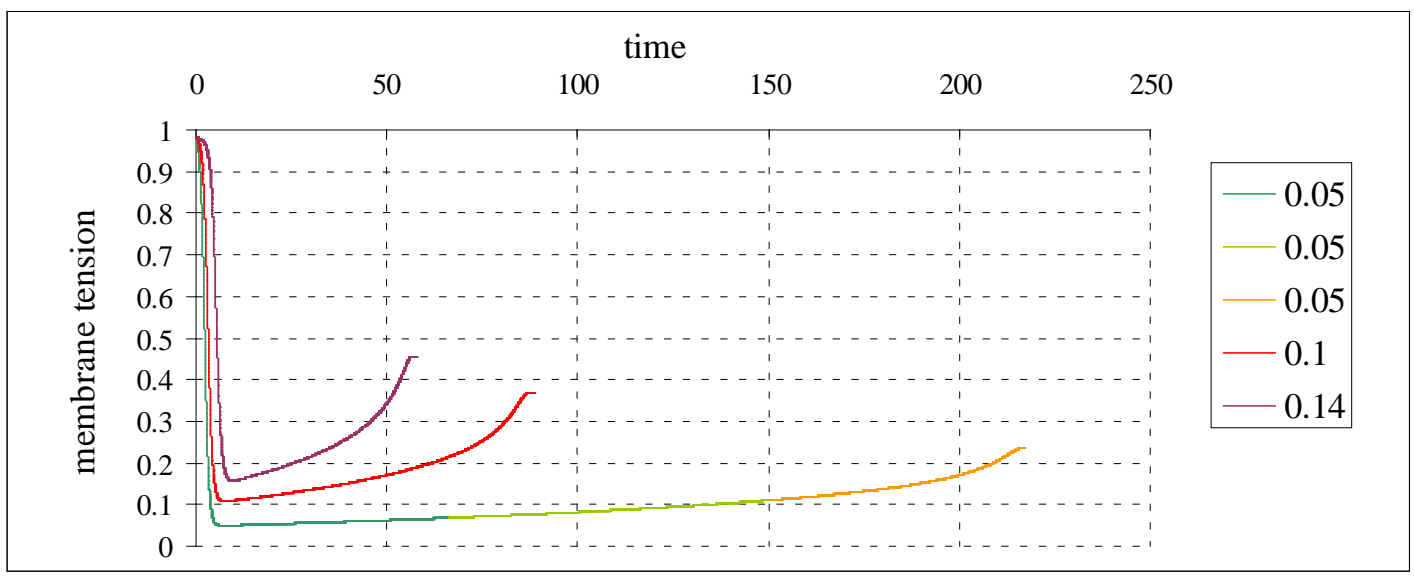

(d)

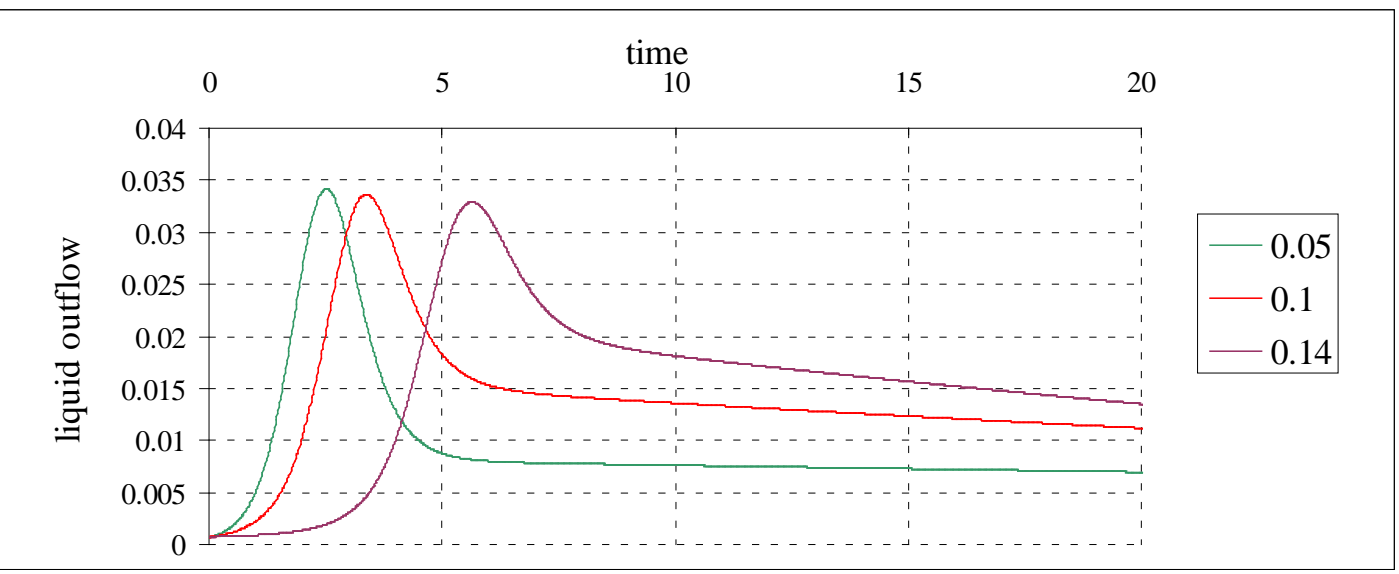




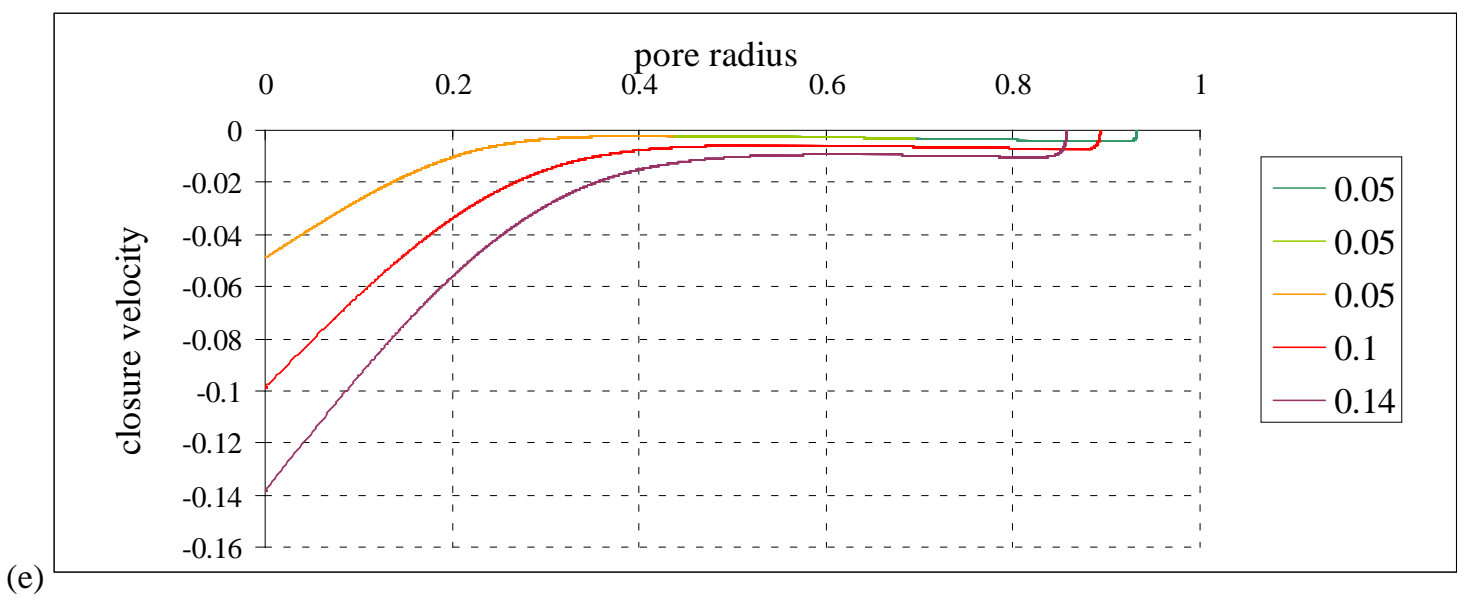

Fig. 4 : Influence of line tension - numerical simulations of Eqs. (5', 6') for constant leak-out parameter $\tilde{r}_{L}=5$ and three different line tensions $\tilde{\mathfrak{I}}=0.05-0.1-0.14$; (a) pore radius $\tilde{r}$ vs. time $\tilde{t}$; (b) drop of vesicle radius $\Delta$ vs. time $\tilde{t}$; (c) surface tension $\tilde{\sigma}$ vs. time $\tilde{t}$; (d) liquid outflow $Q$ in the early times; (e) closure velocity $\dot{\tilde{r}}<0$ vs. pore radius $\tilde{r}$.

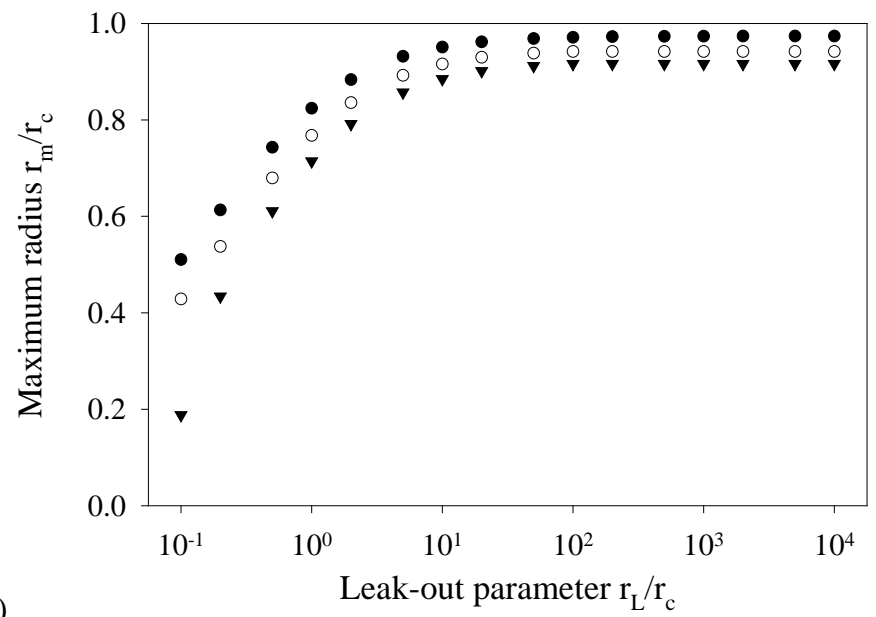

(a)

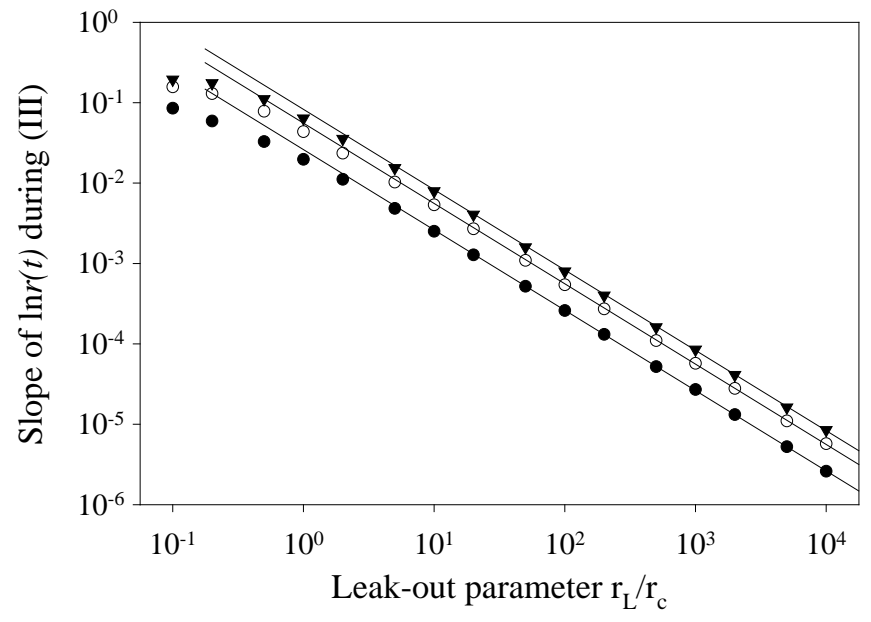


Fig. 5: Scaling laws - the leak-out parameter $\tilde{r}_{L}$ is varied over a broad range, and three values of the line tension $\widetilde{\mathfrak{I}}$ are studied : 0.05 (closed circles), 0.1 (open circles) and 0.14 (closed triangles); (a) maximum pore radius $\tilde{r}_{m}$ vs. $\tilde{r}_{L}$; (b) closure velocity during regime (III) (slope of semilogarithmic plot $\ln \tilde{r}(\tilde{t})$ vs. $\tilde{t})$ vs. $\tilde{r}_{L}$.

(a)
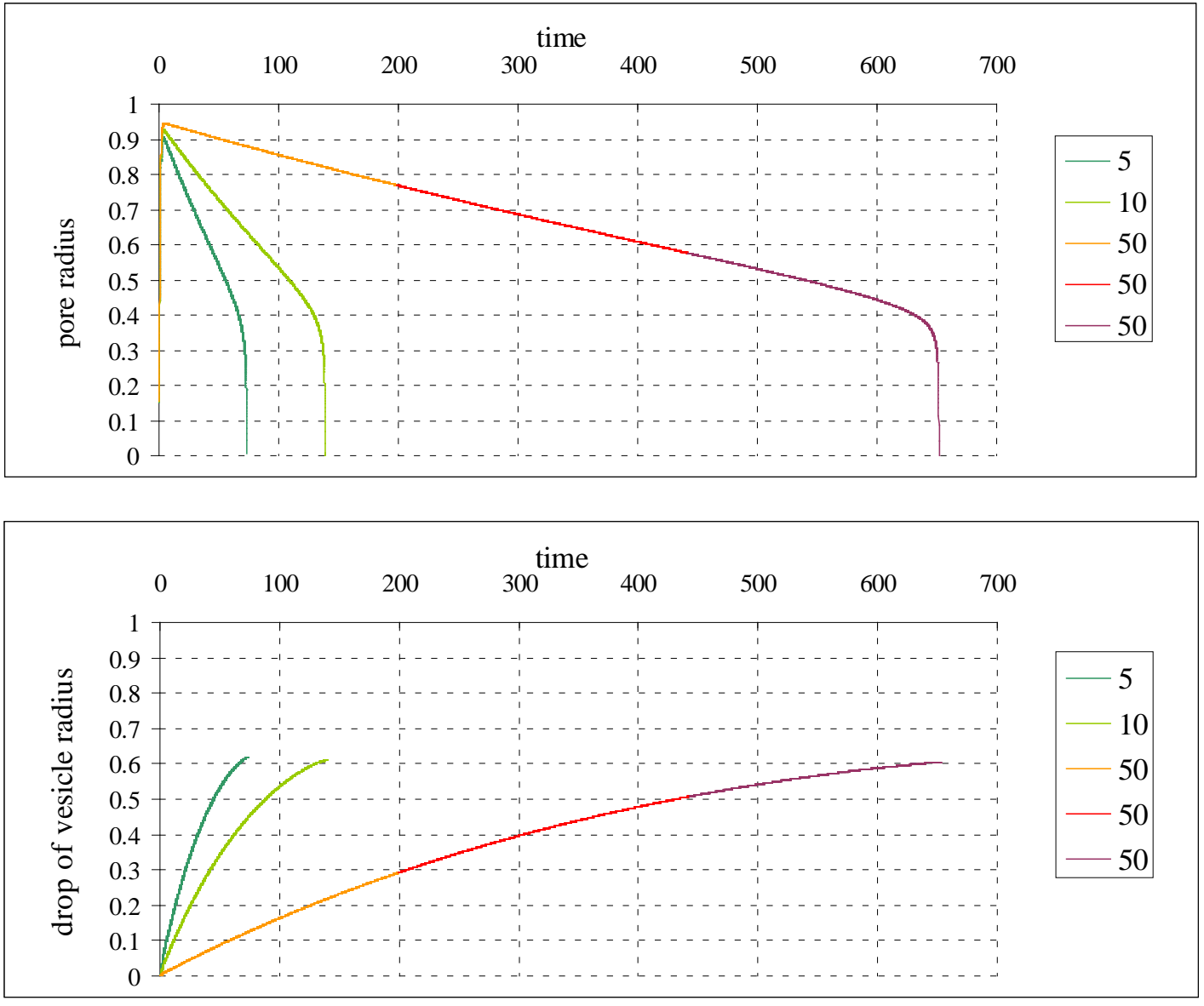

(b)

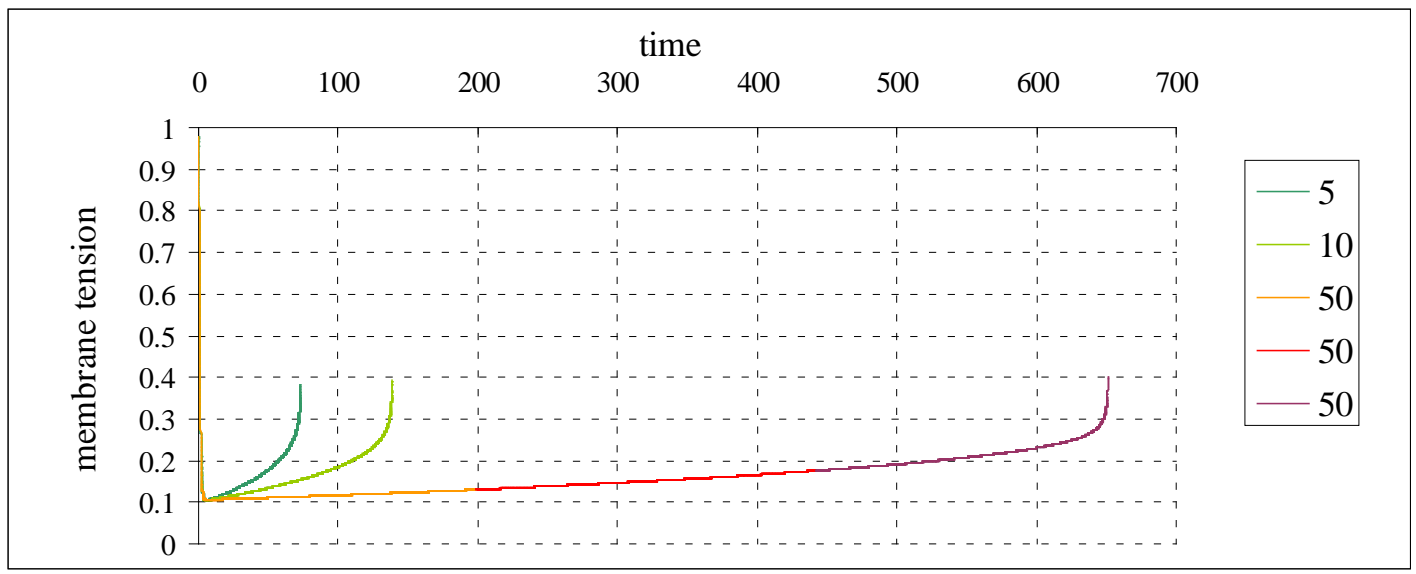

(c) 
(d)

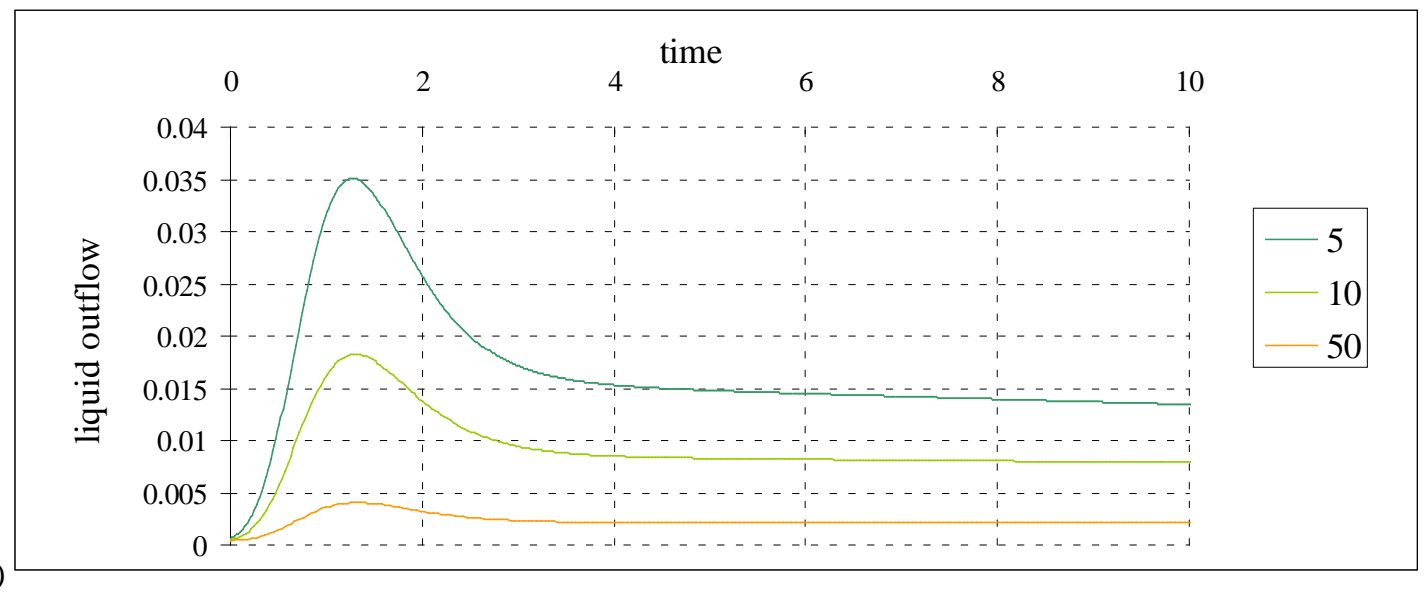

(e)
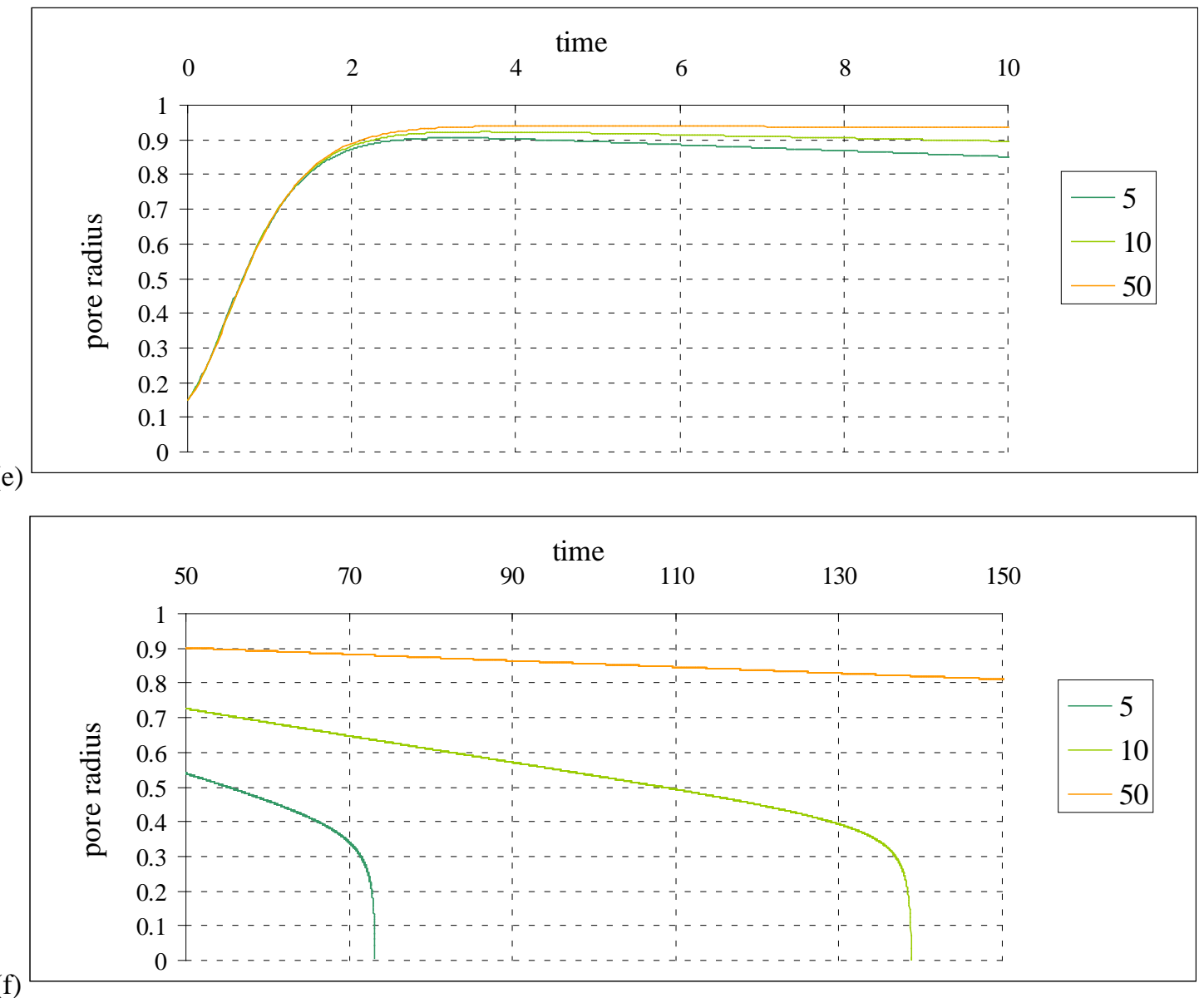

Fig. 6: Dissipation dominated by backflows - numerical simulations of Eqs. (A5) for constant line tension $\widetilde{\mathfrak{I}}=0.1$ and different values of the leak-out parameter $r_{L}{ }^{\prime}=5-10-50$; (a) pore radius $\tilde{r}$ vs. time $\tilde{t}$; (b) drop of vesicle radius $\Delta$ vs. time $\tilde{t}$; (c) surface tension $\tilde{\sigma}$ vs. time $\tilde{t}$; (d) liquid outflow $Q$ in the early times; (e) enlarged view of the growth (I); (f) enlarged view of the final stage (IV). 